UCRL-ID-125270

\title{
Report on Demonstration Project: Imaging Detection of Unexploded Ordnance using Giant Magnetoresistive Sensor Arrays
}

Alison Chaiken

September 1996

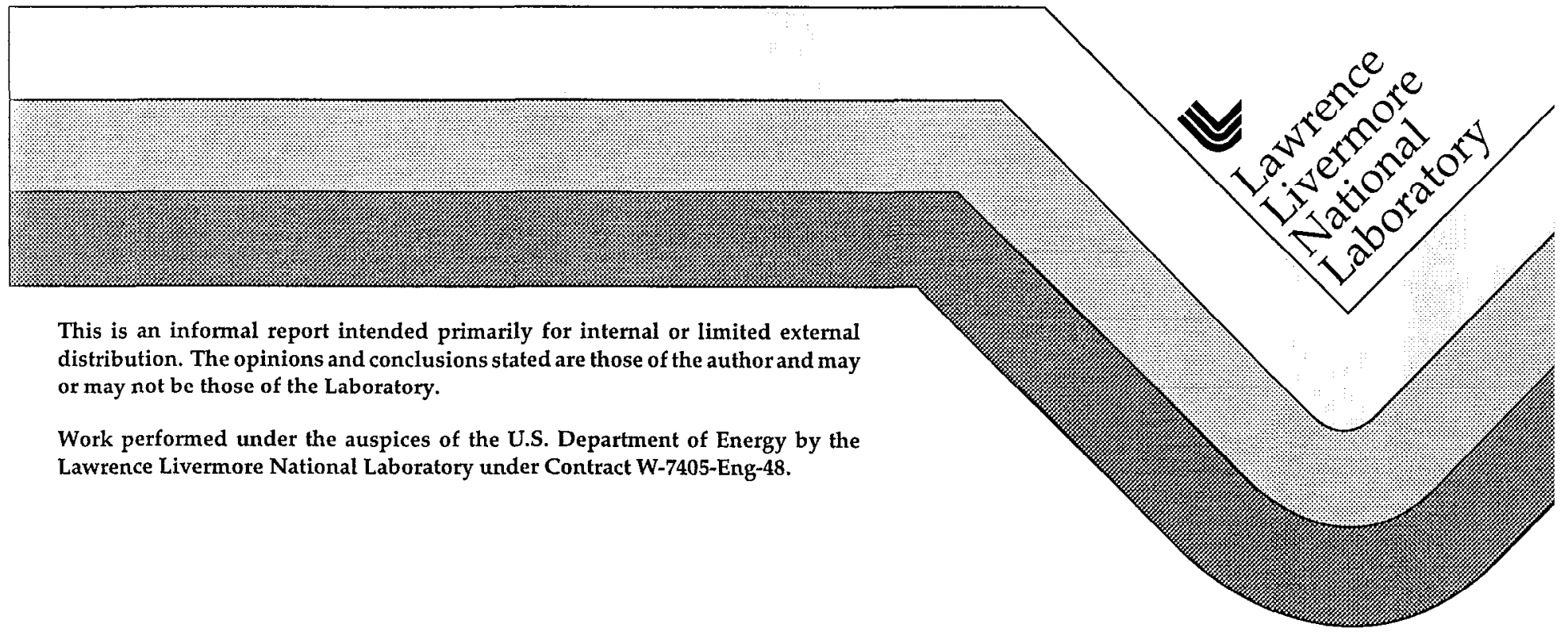




\section{DISCLAMMR}

This document was prepared as an account of work sponsored by an agency of the United States Government. Neither the United States Government nor the University of California nor any of their employees, makes any warranty, express or implied, or assumes any legal liability or responsibility for the accuracy, completeness, or usefulness of any information, apparatus, product, or process disclosed, or represents that its use would not infringe privately owned rights. Reference herein to any specific commercial product, process, or service by trade name, trademark, manufacturer, or otherwise, does not necessarily constitute or imply its endorsement, recommendation, or favoring by the United States Government or the University of California. The views and opinions of authors expressed herein do not necessarily state or reflect those of the United States Government or the University of California, and shall not be used for advertising or product endorsement purposes.

This report has been reproduced directly from the best available copy.

Available to DOE and DOE contractors from the Office of Scientific and Technical Information

P.O. Box 62, Oak Ridge, TN 37831

Prices available from (423) 576-8401

Available to the public from the National Technical Information Service

U.S. Department of Commerce 5285 Port Royal Rd.,

Springfield, VA 22161 


\title{
Report on Demonstration Project: Imaging Detection of Unexploded Ordnance using Giant Magnetoresistive Sensor Arrays
}

\author{
Alison Chaiken \\ Mailstop L-350 \\ Materials Science and Technology Division \\ Lawrence Livermore National Lab \\ Livermore, CA 94550 \\ chaiken@llnl.gov \\ (510) $422-7129$
}

\begin{abstract}
The goal of the project was to demonstrate the feasibility of the detection of buried unexploded ordnance (UXO) using giant magnetoresistive (GMR) sensor arrays. $3 \times 3$ and $5 \times 5$ arrays of off-the-shelf GMR sensors were purchased from Nonvolatile Electronics (NVE) and were interfaced with a data acquisition card and a personal computer. Magnetic images were obtained from a number of ferrous objects, such as threadstock, bolts, and rebar. These images can be interpreted in terms of the remanent magnetic state of the objects. The ability of the GMR sensor approach to discriminate among magnetic objects is assessed and the design of a more realistic UXO detection system is discussed.
\end{abstract}

Introduction: Many techniques are in use or have been proposed for use as UXO detectors. The two most commonly employed technologies are electromagnetic induction detection and fluxgate magnetometry. While time-domain analysis of inductive signals has been suggested as a way to differentiate between hazardous and benign types of buried material, neither the induction detector nor the fluxgate magnetometer may be engineered to produce an image of potential UXO objects. The success of imaging technologies based on arrays of detectors like forward-looking infrared cameras for infrared target identification and charge-coupled device video cameras for consumer applications suggests that the sensor-array paradigm is worth exploring for UXO detection as well. Neither the electromagnetic induction nor fluxgate magnetometry methods is well-suited for incorporation into a detector array since these sensors are bulky in size. GMR sensors, on the other hand, are now available now in integrated circuit form. These sensors are attractive for a variety of applications because of their high sensitivity (over ten times greater than Hall sensors), 
room-temperature operation (unlike SQUID magnetometers), radiation hardness and moderate cost (currently $\$ 5$ apiece in small quantities).

GMR sensors have never been tested specifically as UXO detectors but are known to have comparable sensitivity to electromagnetic induction systems, although not the extreme sensitivity of cesium-vapor magnetometer systems. A practical UXO system in the end is expected to incorporate a variety of sensor types integrated into a single package so that both maximum sensitivity and imaging capability will be available to operate in concert. The impact of GMR array UXO detectors on DOD site remediation activities is potentially great. The inspection of false positives during cleanup of contaminated areas adds greatly to the cost and duration of site remediation. Typically, 50 to 60 pounds of scrap metal are recovered for each ordnance item found using present technology. An easy-to-use imaging UXO detection system would allow a relatively inexperienced user to rapidly distinguish between buried ordnance and other ferrous objects. Possible follow-on work could allow extension of the GMR imaging technology to the detection of non-ferrous objects or to non-destructive testing applications. Successful development of an imaging detector for site remediation will provide useful baseline information for design of a battlefield-deployable UXO imaging system.

System Design: Nonvolatile Electronics' NVS5B15 sensors were employed for this project. The data sheet for the NVS family of GMR Magnetic Bridge Sensors is appended at the end of this report. NVE is at present the only commercial vendor of GMR sensors, although other electronics companies (for example, Honeywell and Motorola) are expected to offer GMR products in the next few years.

GMR sensors detect a single vector component of an applied field, like Hall generators or pulsed inductive detectors, so three orthogonal sensors banks will be necessary for full 3D imaging capability. A single 2D array of sensors was selected for this demonstration where alternate sensors have orthogonal axes of sensitivity. The checkerboard layout of the $5 \times 5$ array of sensors is illustrated in Figure 1 and 2. In order to simplify interpretation of the magnetic images at the end of the report, the outputs from the sensors with vertical and horizontal axes of sensitivity are displayed separately, as illustrated on the right side of the drawing. The performance of the $5 \times 5$ sensor array has been compared with a $3 \times 3$ array (not shown) where all the elements have the same vertical axis of sensitivity. The $3 \times 3$ array uses the same printed circuit 
board layout as the $5 \times 5$ so that the effect of varying the sensor spacing by a factor of two could be determined.

A schematic of the imaging system is shown in Figure 3. The sensor arrays were interfaced with an electronics chassis that contained a 15 and $5 \mathrm{~V}$ power supply. The outputs of the sensors were connected to a National Instruments 64-channel data acquisition card which was installed in a Pentium PC. National Instruments' LabView software was used to acquire the images that follow. The images are unprocessed beyond resizing and adjustment of the color scale for printability. Since there are only 25 sensors per image, the data files are only about 400 bytes in size. Each image is an average of 1000 readouts of the full array during a 10-second period (acquisition rate $=$ $100 \mathrm{~Hz}$ ), although there is no reason that data could not be acquired much more rapidly (perhaps $10 \mathrm{kHz}$ ). 1000 readouts of the array was decidedly overkill; images were not degraded by the averaging of smaller data sets.

A variety of ferrous objects were imaged. These included tools, bolts, nails, rebar and permanent magnets. All objects were imaged in their remanent magnetic state (i.e., no external applied field) except where otherwise specifically noted. Before an image was acquired, the no-object output of all the sensors was obtained using the PC. This background signal represents a combination of offsets in the sensors, the sensors' response to the earth's field (no magnetic shielding was used) and their response to magnetic objects in the laboratory where the data was acquired, e.g. furniture and rebar in the floor. This background signal was saved to a file and then subtracted from subsequent data. Objects to be imaged were placed typically $1.5 \mathrm{~cm}$ above the sensor array on a lexan stand. The falloff of the signal from the array with separation was studied by placing firebricks between the array and the ferrous object. Larger objects such as rebar could be detected at a meter separation (signal:background ratio of 2:1) although there was no real image at that separation with the $12 \mathrm{~cm} \times 12 \mathrm{~cm}$ array used for this demonstration.

Results: A sampling of images produced with the GMR sensor array is shown in Figures 4-10. Figure 4 shows an image of a $\# 10$ threaded rod $1.5 \mathrm{~cm}$ above the array, as pictured in the top-view drawing on the right. The two blue-scale images on the left are data obtained from the sensor array. The top image shows data from the GMR elements (labelled "corners") with a vertical axis of sensitivity. In the "corners" image, a value of 0 volts is displayed at the positions corresponding to the elements with a 
horizontal axis of sensitivity. The bottom image, labelled "others," shows data from the GMR elements witha horizontal axis of sensitivity. In the "others" image, the positions corresponding to the "corners" elements are displayed as 0 volts. Comparison with Figure 1 will clarify which pixels are meaningful in the two images. Clearly in a real UXO detection system, more sophisticated software would combine the two images in a contour or vector plot. Here darker blue colors indicate higher magnetic flux, while grayer blue colors indicate lower magnetic flux. The color scheme is illustrated by the scale on the right of each image. The numbers next to the color scale are the sensor signal in volts, so that $1.4 \mathrm{e}-2$ means $140 \mathrm{mV}$ of signal.

In the top image of Figure 4, the magnetic poles on the ends of the rod are being picked up by the sensors at the upper right and lower left corners. In the bottom image, the sensors are responding to magnetic flux leaking from the sides of the rod. While the pixel in the upper left-hand corner of the top image is confusing, the general shape and size of the rod can be determined through examination of the image.

Figure 5 shows another image of the same rod, only this time flipped over so that it is pointing towards the opposite corners of the array. The movement of the magnetic poles to the upper left and lower right corners is obvious in both images. There are several reasons why this image is not a perfect mirror of Figure 4, namely different lateral placement of the rod on the array and different rotation of the rod about its own axis. The magnetic domains in the rod may not be azimuthally symmetric, with the result that the image may depend somewhat on which side of the rod is facing downward. The rod in Figure 5 is also oriented differently with respect to the earth's field than in Figure 4. In a real UXO system possible confusion created by different remanent states of objects can be addressed through application of a pulsed magnetic field, as is done with present-day electromagnetic induction detectors. This is discussed further below.

Figure 6 now shows an image of the same rod in a 6 Oe external applied field which was generated with air-core Helmholtz coils. Before acquiring the image, the background of the array was characterized in the presence of the 6 Oe field. The most striking part about this image is that it looks much like Figure 4, showing that the GMR sensor array is able to image ferrous objects even in the presence of a reasonably substantial background magnetic field. The reason for the similarity of the two images is that the array detects magnetic inhomogeneities, not the scalar magnetic field 
amplitude like a cesium-vapor magnetometer. There is reason to believe that arraybased detectors will usable with magnetic soils such as those found on the formal naval bombing range on the Hawaiian island of Kahoolawe as long as the soils are reasonably homogeneous on the length scale of the objects to be detected.

Another point about Figure 6 is that the image is a bit clearer than in Figure 4, where no external field is applied. The improved image quality occurs because much of the flux from the applied field passes through the magnetically soft rod. A more complete outline of the rod could be made by acquiring another image with an applied field in the orthogonal in-plane direction. In fact, a real UXO system would likely incorporate 3 sensor arrays, each with a different orthogonal axis of sensitivity. Data would be read out from each array while a coil applying a magnetic field along that direction is energized. When fully realized such a system would incorporate a rotating magnetic field and synchronous acquisition from the 3 orthogonal arrays. An additional group of 3 sensors could be used with a portable GMR detector to eliminate noise due to motion of the elements in the earth's magnetic field. (Such a noise-elimination scheme has recently been described for a fluxgate magnetometer system by Allen, Koch and Keefe. See the UXO Forum 1996 Conference Proceedings, p. 1.)

Figure 7 illustrates the ability of the array to image slightly more complex objects. Here two bolts have been placed $1.5 \mathrm{~cm}$ above the array. The "corners" image shows substantial flux from the threaded part of the bolt, while the "others" image shows a more difficult to interpret pattern of flux possibly arising from complex domain patterns in the bolt head. The overall " $\mathrm{V}$ " symmetry of the objects is apparent in both images. The image of the two bolts would be greatly improved by application of an external field and by a higher resolution array, with more pixels on each object.

Figures 8 and 9 show the response of the array to a more massive object, a piece of 3/4"diameter rebar rod. Figure 8 was acquired with a $1.5-\mathrm{cm}$ rod-array separation, while the separation for Figure 9 is $9 \mathrm{~cm}$. The amount of flux emanating from the rod is quite large, lighting up most of the pixels in Figure 8. The two images of Figure 8 together show that we have a large object with a vertical axis of symmetry which is coming down from the top of the image. Comparing Figure 8 to Figure 9, we see that the maximum signal has been reduced from $280 \mathrm{mV}$ to $210 \mathrm{mV}$, still well above the typical background level of 20-50 $\mu \mathrm{V}$. The "others" image of Figure 9 looks much like that of Figure 8 , but the "corners" image look substantially different. In part this may be due to 
a different position of the rebar, as discussed with respect to Figures 4 and 5 . However, much of the difference between Figures 8 and 9 is likely due to different spatial falloff of the various components of the magnetization pattern. One must keep in mind that the magnetic field emanating from an object can vary in all three dimensions, and there is no particular reason for the symmetry of a 2D slice taken at one height to be exactly the same as a 2D slice taken at another height. On the other hand, the images sometimes appear rather simple, as in Figure 4. Intelligent synthesis of data and interpretation of images will be the major challenge in building a useful GMRbased UXO detector, although the intrinsic difficulty is not greater than in time-domain analysis of pulsed electromagnetic induction data, for example. In order to capitalize on what is already known about magnetic detection of mines, in a previous proposal to DOD's SERDP program a team was formed that includes Geometrics, Inc. and Arete Engineering Technology Center (AETC) as well as Nonvolatile Electronics and LL.VL.

Several other issues were investigated that are not illustrated by the images reproduced here. One question of interest was whether crosstalk between the elements would cause reduced sensitivity in the $5 \times 5$ array as compared to the $3 \times 3$ array. Crosstalk did not seem to be a problem, although a more careful study with different element spacings is merited as part of a serious array design effort. Another important question is how the array performance depends on the biasing power supply voltage and current. The higher power level $(15 \mathrm{~V})$ did improve performance of the sensors when an external field is applied, but there was no apparent difference in performance in the absence of an external field. Therefore all images above were acquired using the $5 \mathrm{~V}$ bias level, except Figure 6, where the supply voltage was $15 \mathrm{~V}$.

Realization of a fieldable UXO detection system: There are several obvious improvements that would be necessary for a real-world UXO detector. For example, there are questions about portability and ruggedness of a fieldable GMR array system. Since these issues are similar for GMR technology as for other sensing methods, they need not be elaborated on further here, although it is worth noting that the power usage per GMR sensor (about $5 \mathrm{~mW}$ dc for the NVS5B15) is quite reasonable.

It should be clear from examination of the images that having a larger array with additional sensors will produce a more immediatley recognizable result. There are no serious practical problems with constructing a larger array. Ideally the individual elements of a large array would be addressable via row and column transistors, much 
like a random-access memory or charge-coupled device array. Since UXO objects tend to be many $\mathrm{cm}$ in extent, the GMR elements can be spaced far enough apart that there is plenty of printed-circuit board area available for these other electronic components.

For this demonstration, no signal conditioning electronics were employed; the sensors are wired directly to the data acquision card. A portable system with integrated fieldproducing coils and 3-axis sensitivity will require considerably more sophisticated signal conditioning and processing electronics. Since signal levels, data rates, and data amounts are all moderate for this application, design of the support electronics for a GMR detector should not be straightforward. The implementation of UXO-recognition software is more ambitious since magnetic pattern recognition for extended objects is still a new field. Geometrics, AETC and Conductus Corporation of Sunnyvale, CA are engaged in development of a magnetic imaging system using cryogenic SQUID magnetometer elements. Some of their experience with analysis of signals from SQUID systems may be transferable to GMR systems.

Finally it is worth noting that NVE's NVS sensors are the very first GMR-based products to be commercially available; GMR was only discovered in 1988. More sensitive GMR elements are expected to be available later this year, with substantial improvement possible in the near future.

Part of this work was performed under the auspices of the U.S. Department of Energy by Lawrence Livermore National Laboratory under contract number W-7405-ENG-48. Thanks are due to Gary Johnson and Alan Wiltse of LLNL and John Anderson and Russell Beech of NVE for assistance with this project.

\section{Figure Captions}

Figure 1. An illustration of the layout of the 5x5 GMR sensor array. Each white square in the "full array" drawing on the left represents one NVE NVS5B15 sensor. The arrows on this drawing indicate the orientation of the axis of sensitivity for each sensor. The right side of the figure shows how the outputs of the elements are split up in the images of Figures 4-9 according to the direction of their sensitivity axis. 
Figure 2. Actual size layout of the printed circuit board on which the GMR elements are mounted. The orientation of the axis of sensitivity for each element is determined by the way it is soldered to the circuit board.

Figure 3. A schematic of the experimental setup. A ferrous object was placed on a lexan stand (not shown) above the sensor array, which was interfaced to simple readout electronics. The spatial dependence of the output signal from each of the 25 elements was displayed on the screen of the PC. Each data set was split in software into a "corners" and "others" image so that the spatial variation of each of the vector components of magnetic field could be viewed separately.

Figure 4. Image of a threaded \#10 rod placed above the GMR array. The drawing on the right shows an idealized top view of the placement of the rod above the array. On the left, the blue-scale images show the response of the elements to the magnetic field emanating from the rod. The "corners" image shows only data from the elements with a vertical axis of sensitivity, while the "others" image shows data from the elements with a horizontal axis of sensitivity. The outline of the rod is visible where magnetic flux

Figure 5. Same as Figure 4, but with the rod flipped about a horizontal axis. The image is not quite a mirror reversal of Figure 4.

Figure 6. Same as Figure 4, but with a 6 Oe external applied field acting on the rod and the array. The image looks similar to Figure 4 and is even a bit clearer despite the necessary subtraction of the background signal from the external field. This image suggests that GMR arrays will be able to locate ferrous objects even in magnetic soils of volcanic origin.

Figure 7. Image of two ferrous bolts placed above the array. The outline of the bolts is not directly visible, but the symmetry of the pattern is recognizable. More GMR elements and concomitant higher spatial resolution could substantially improve this image.

Figure 8. Image of a rebar rod at $1.5 \mathrm{~cm}$ above the GMR array. Application of an external field would improve this image by simplifying the domain pattern of the rod. The rebar causes a finite signal in most of the elements by virtue of its large size. 
Figure 9. Image of the same rebar rod at $9 \mathrm{~cm}$ above the GMR array. The rod could readily be detected out to 1 meter above the array, although imaging capability is lost once the separation between the object and the array becomes larger than the size of the array. 
Corners

Full array

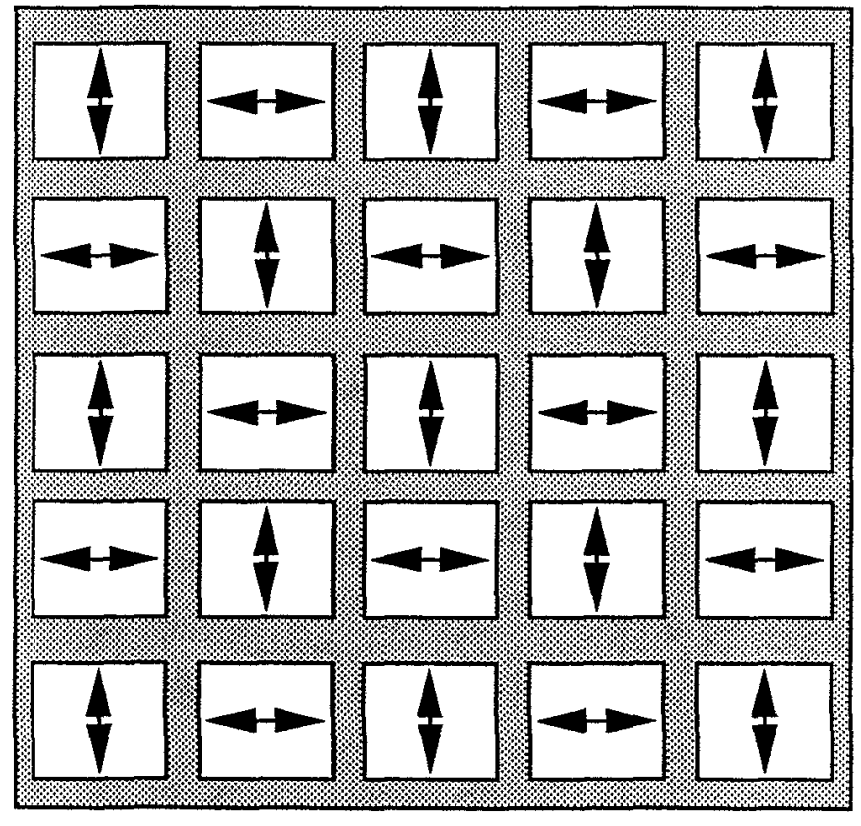

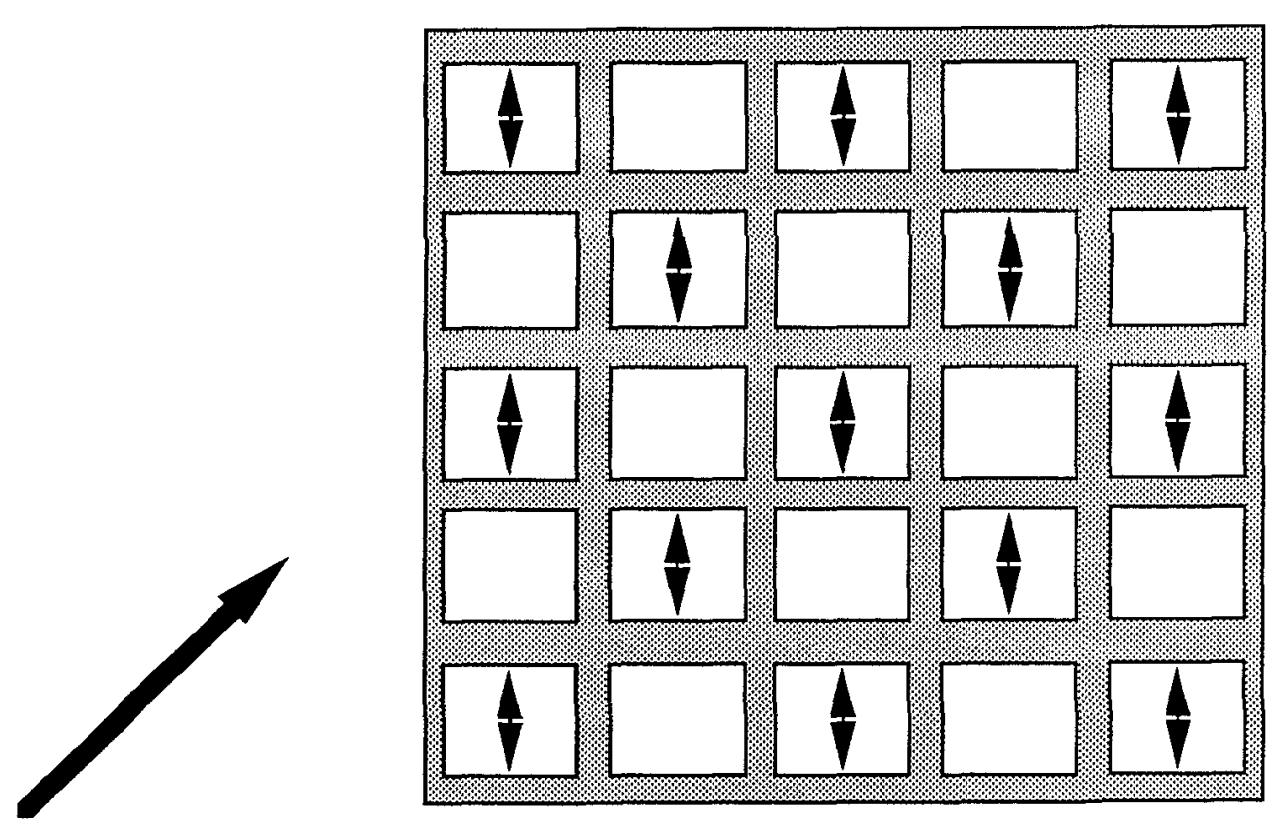

Others

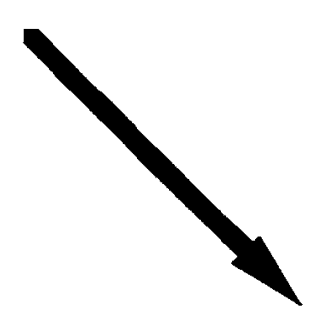

$$
\text { Fignel }
$$

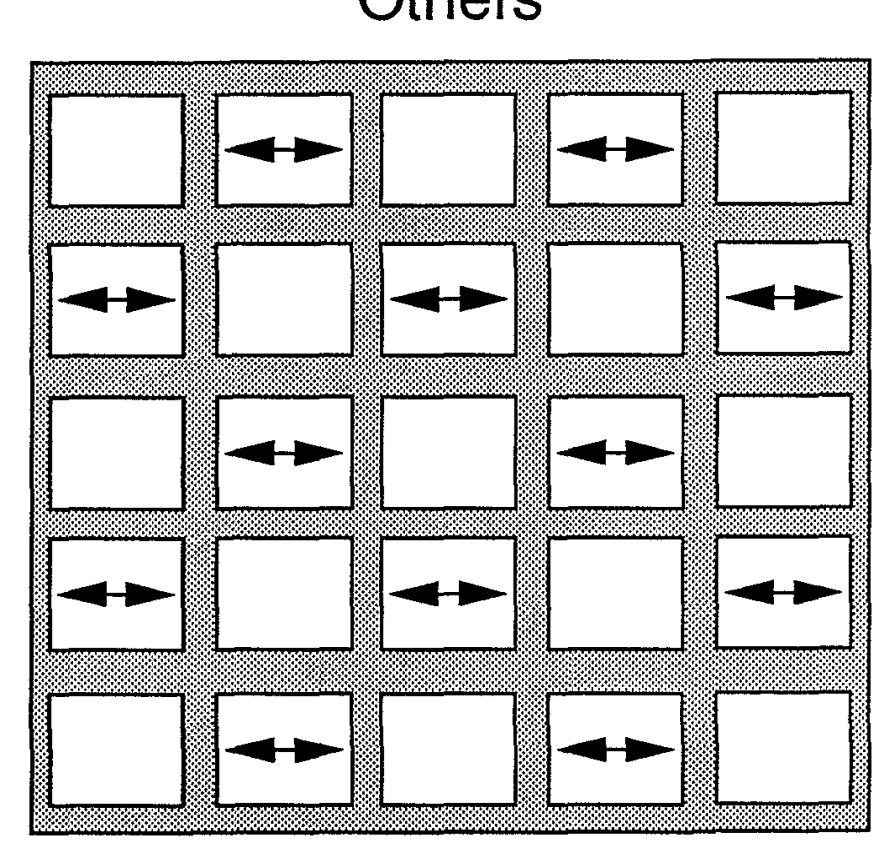




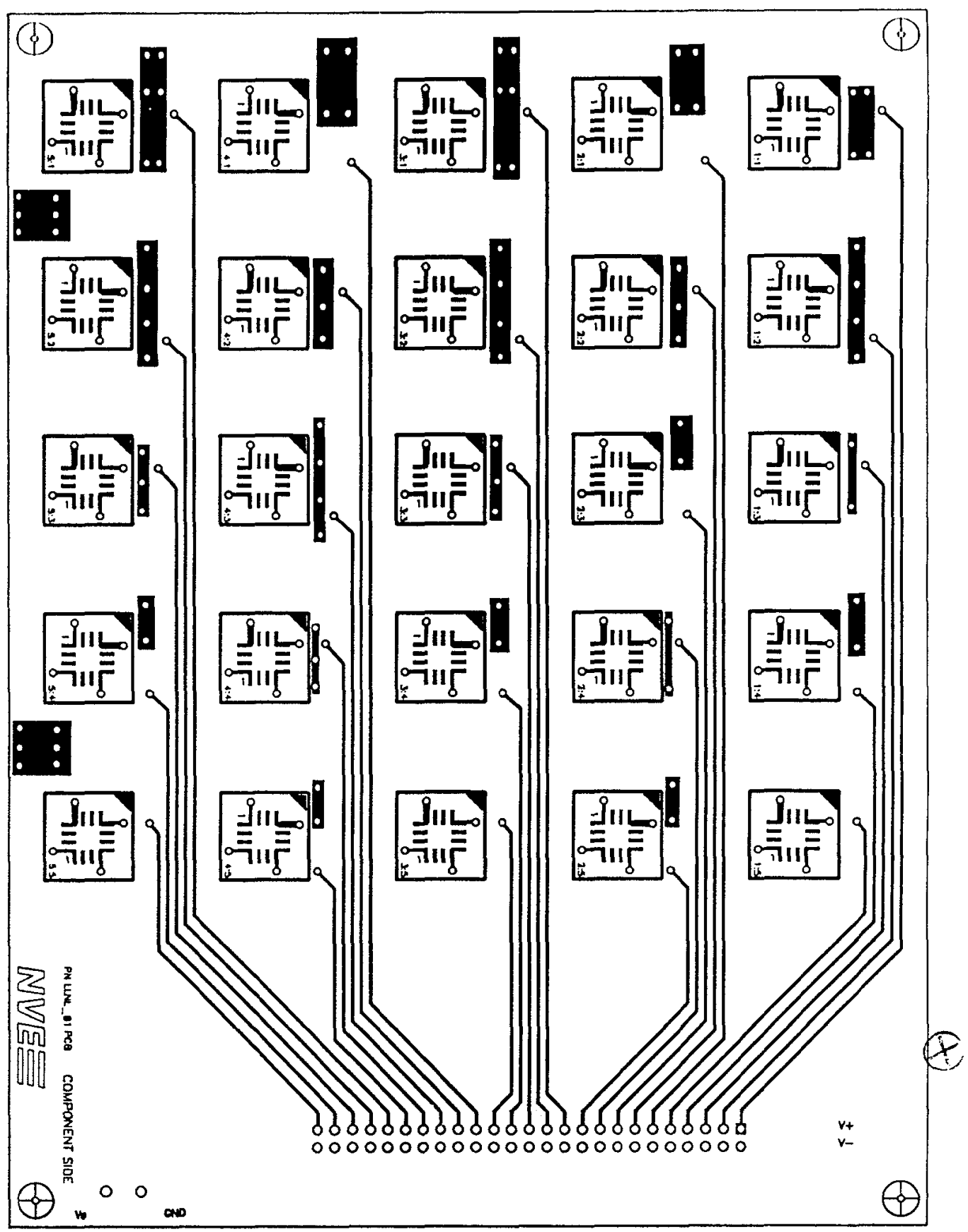




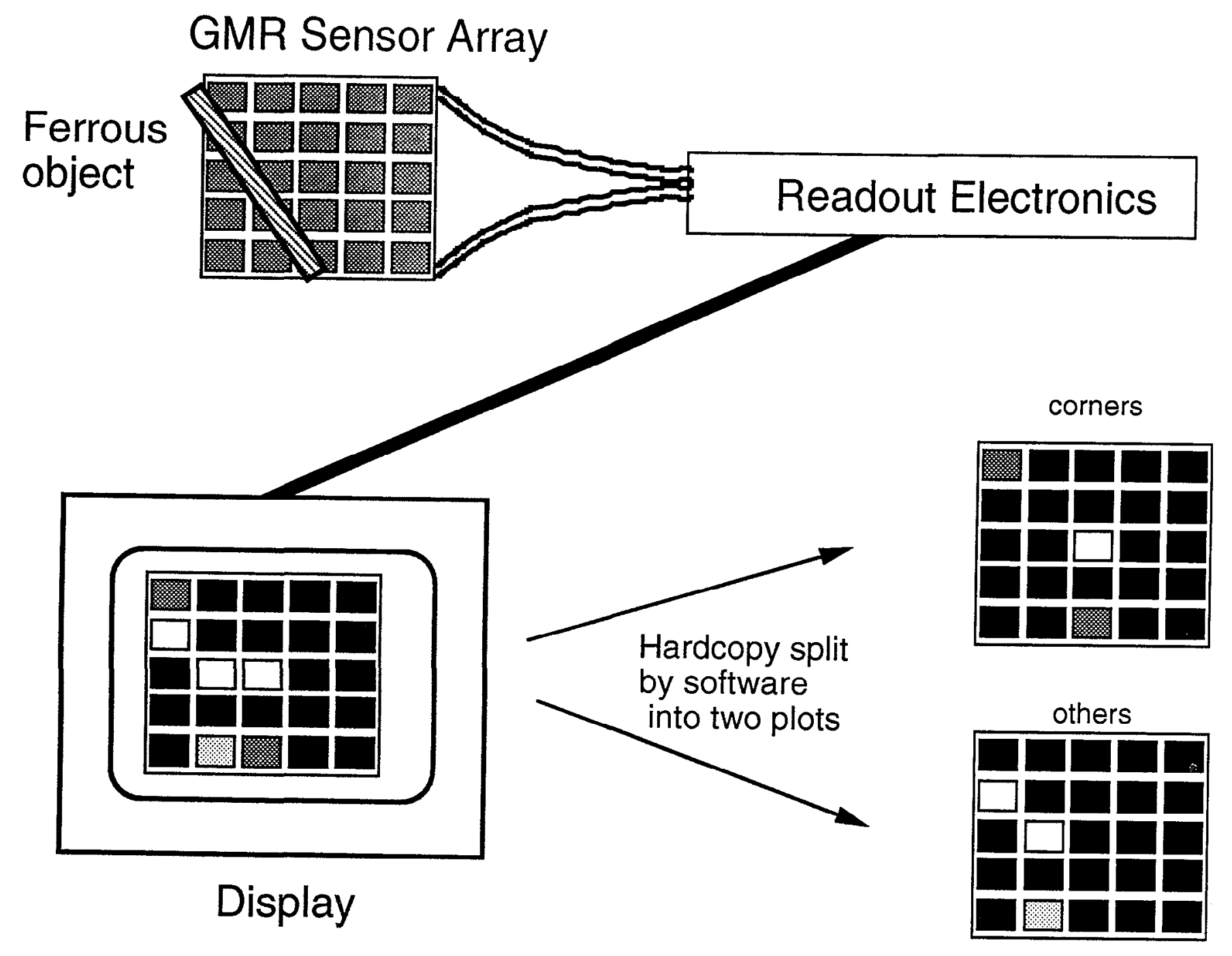




\section{$\underline{\text { Rod above GMR Sensor Array }}$}

Axis of sensitivity ()

Axis of sensitivity

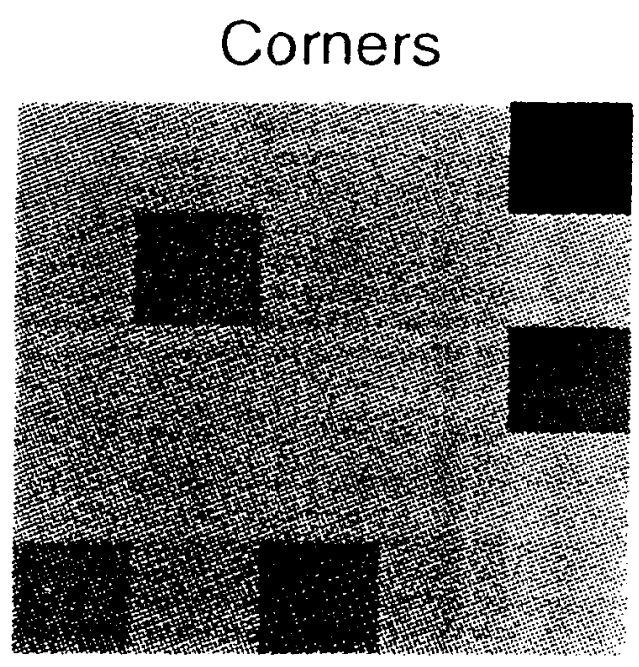

\section{Others}

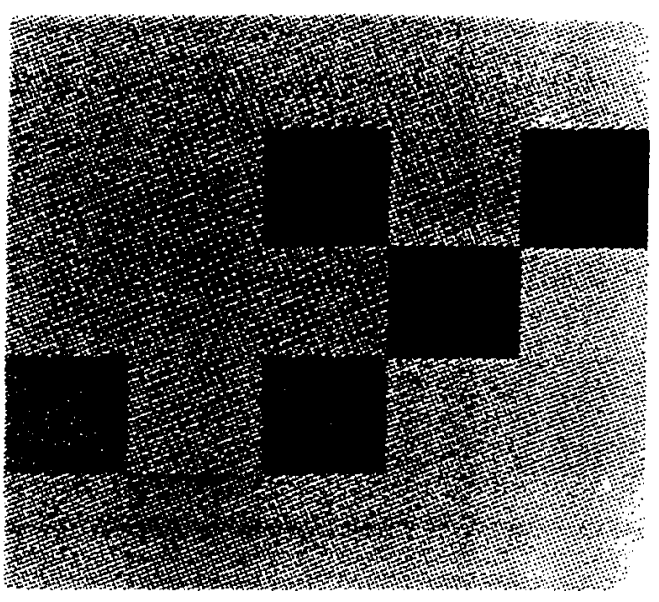

Rod above Sensor Array

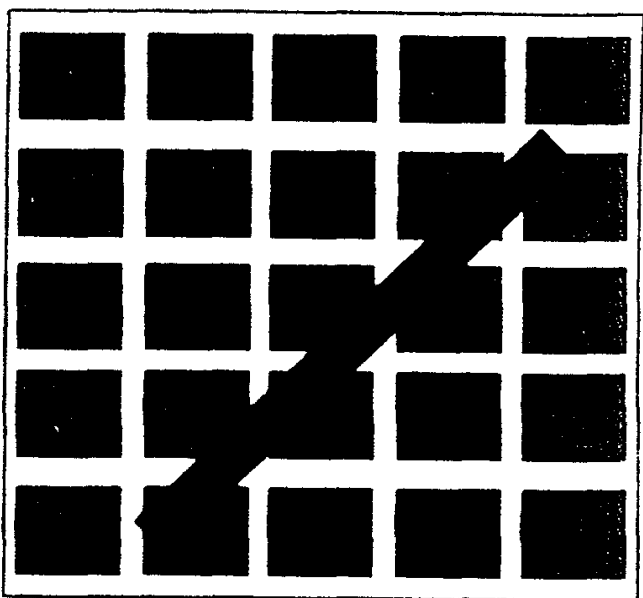

$-1.000 \mathrm{E}-3$

$0.000 \mathrm{E}-0$

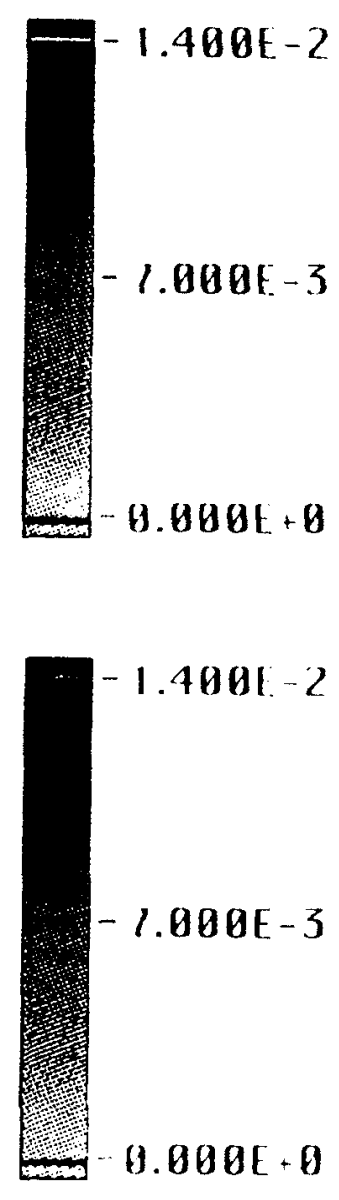




\section{od above GMR Sensor Array}

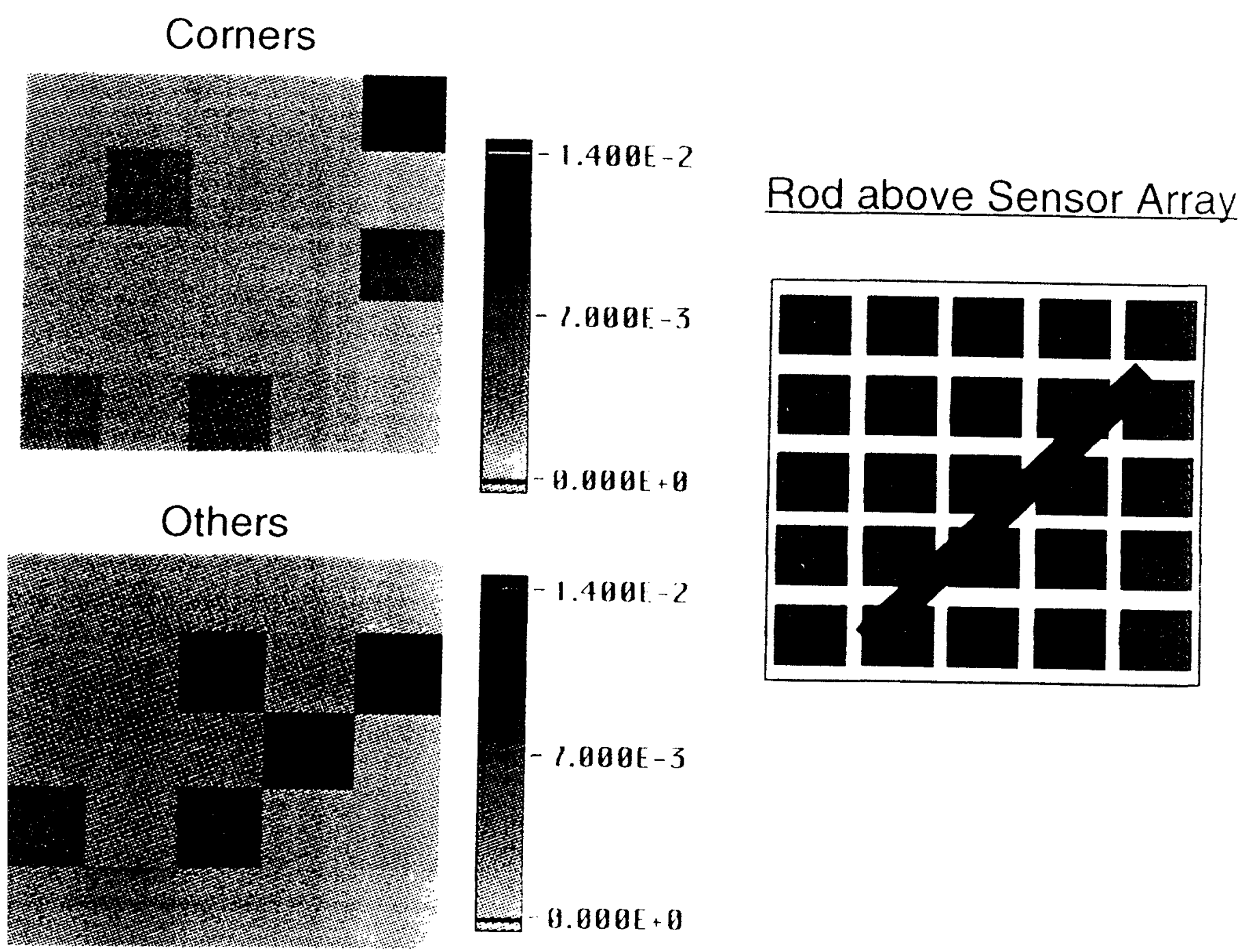




\section{$\underline{\text { Rod above GMR Sensor Array }}$}

Axis of sensitivity

D

Axis of sensitivity

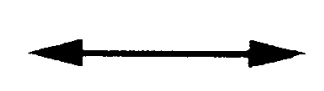

Corners

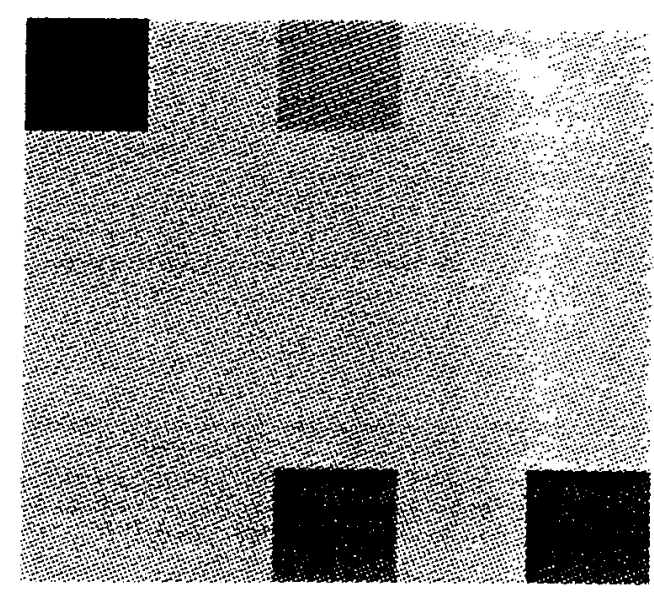

Others

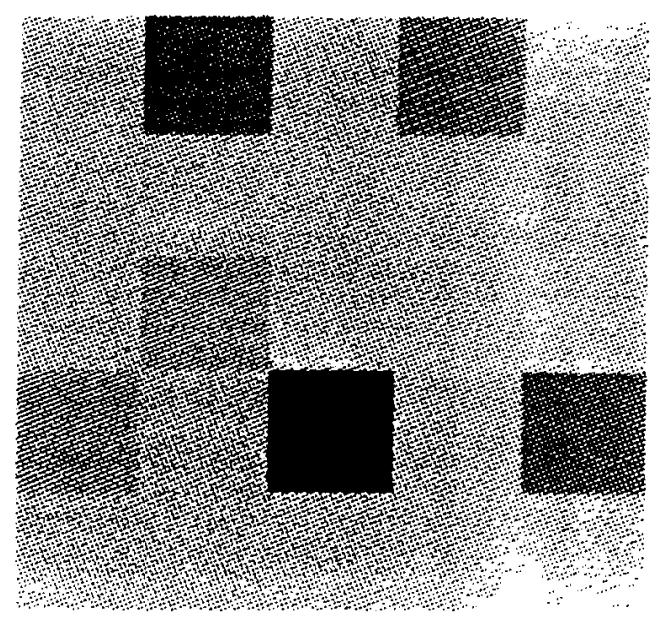

Rod above Sensor Array

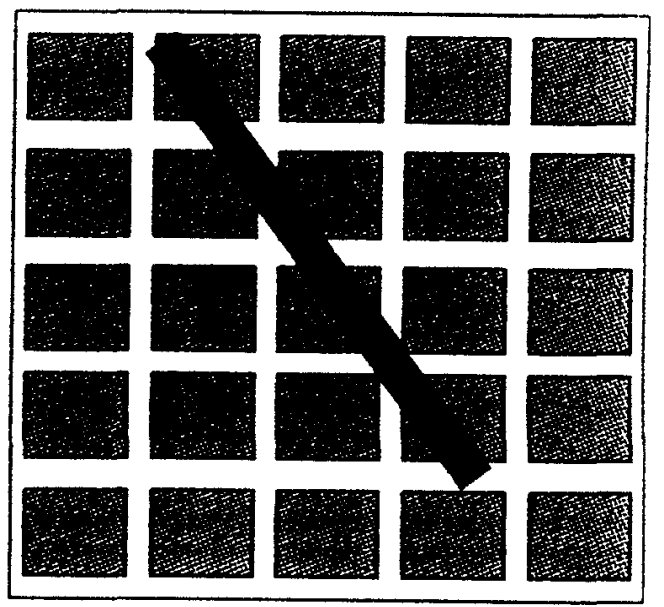




\section{Rod above GMR Sensor Array in a 60) Applied Field}

Axis of sensitivity

$(8)$

$$
4
$$

Axusof sensitivity

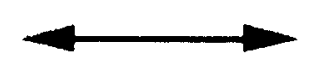

Corners

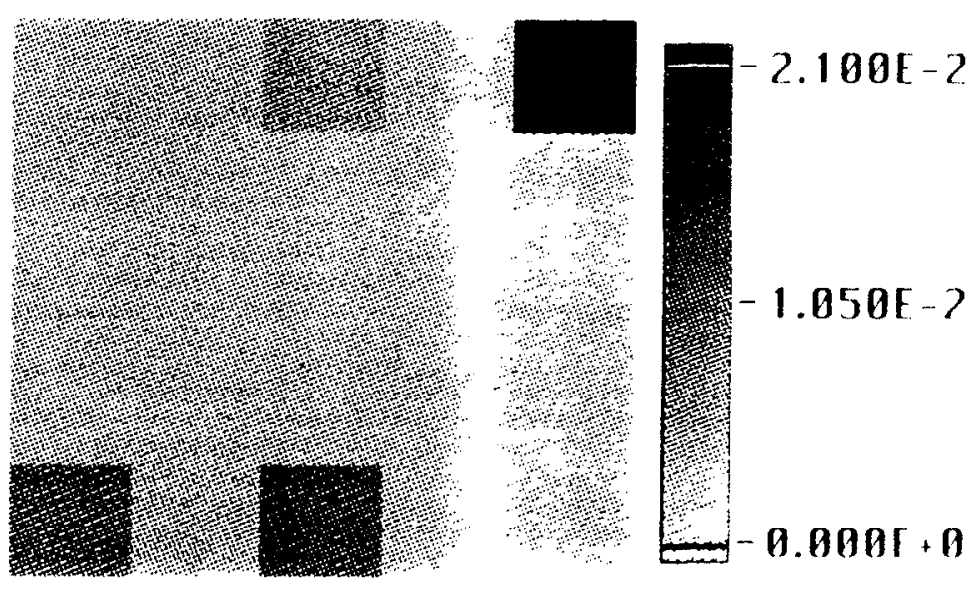

Others

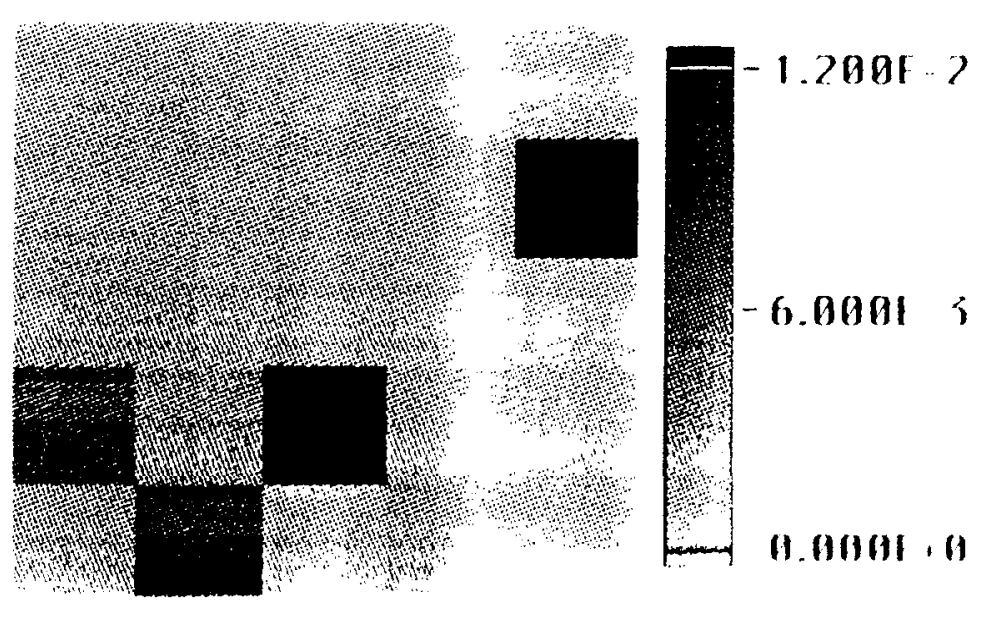

Rod above Sensor Array

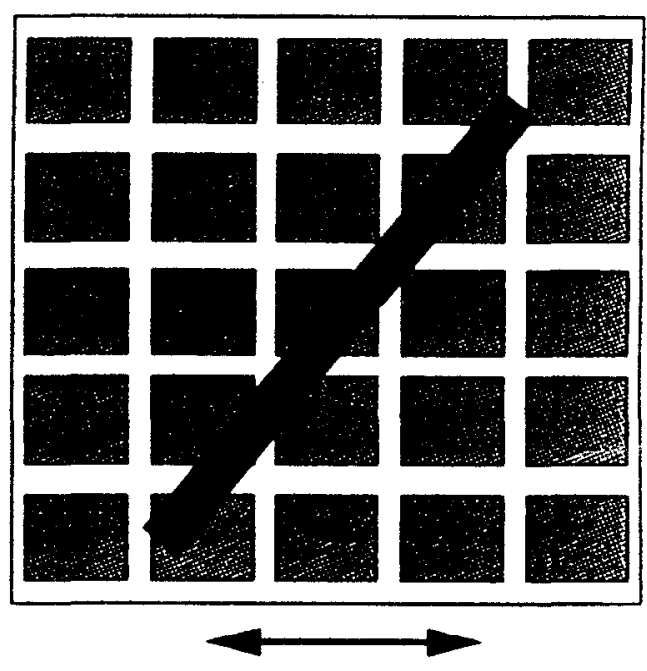

o( ) external applied ficld 


\section{Two Bolts above GMR Sensor Array}

Axis of sensitivity

(马)

Axis of sensitivity

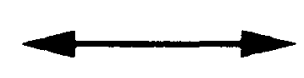

Corners
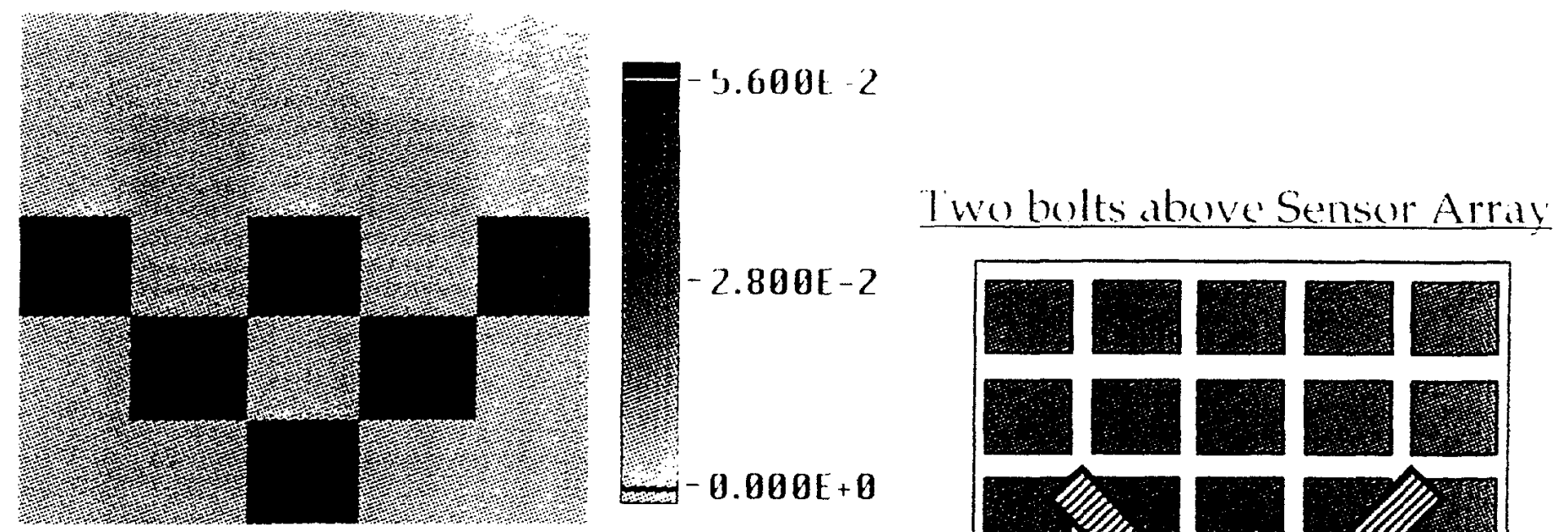

$-2.800 \mathrm{E}-2$

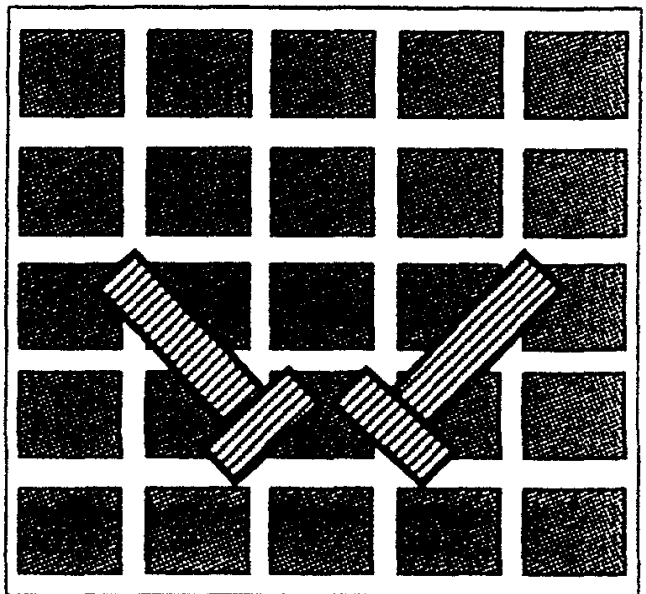

F.gue 


\section{Rebar Rod above GMR Sensor Array}

Axis of sensitivity

+)

Axis of sensitivity

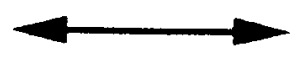

\section{Corners}

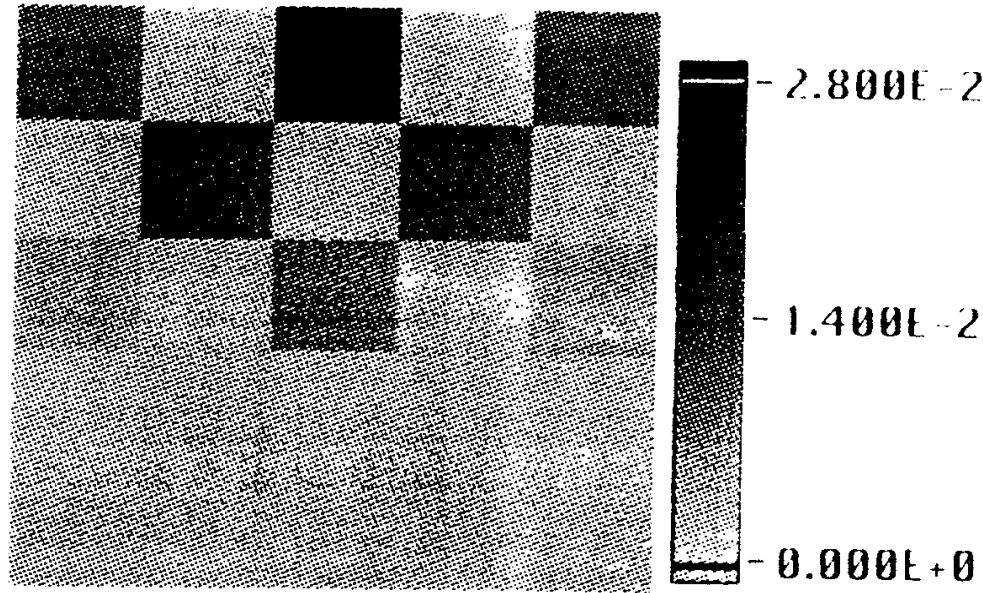

Others

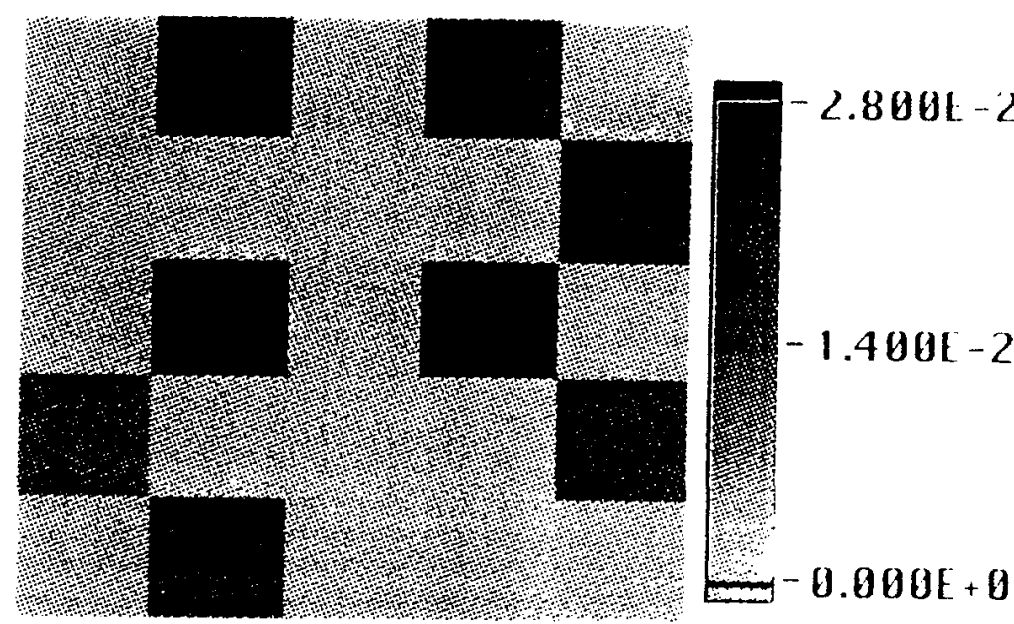

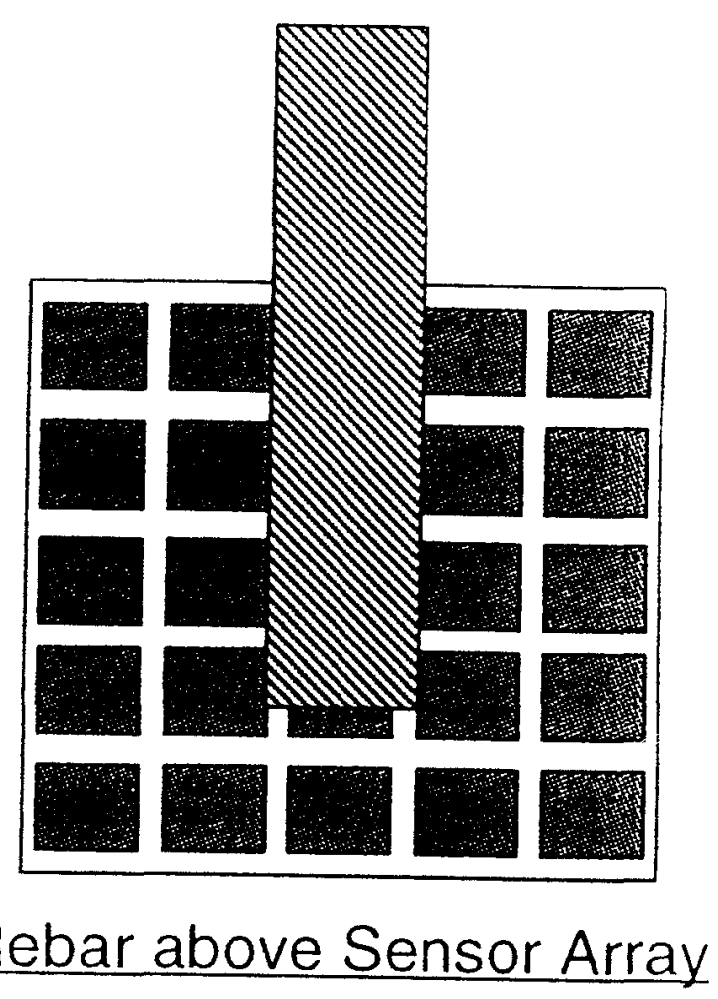

Rebar above Sensor Array 
Rebar Rod above (iMR Sensor Array

AvM of ensitivity

(8)

Axs of sensitivity

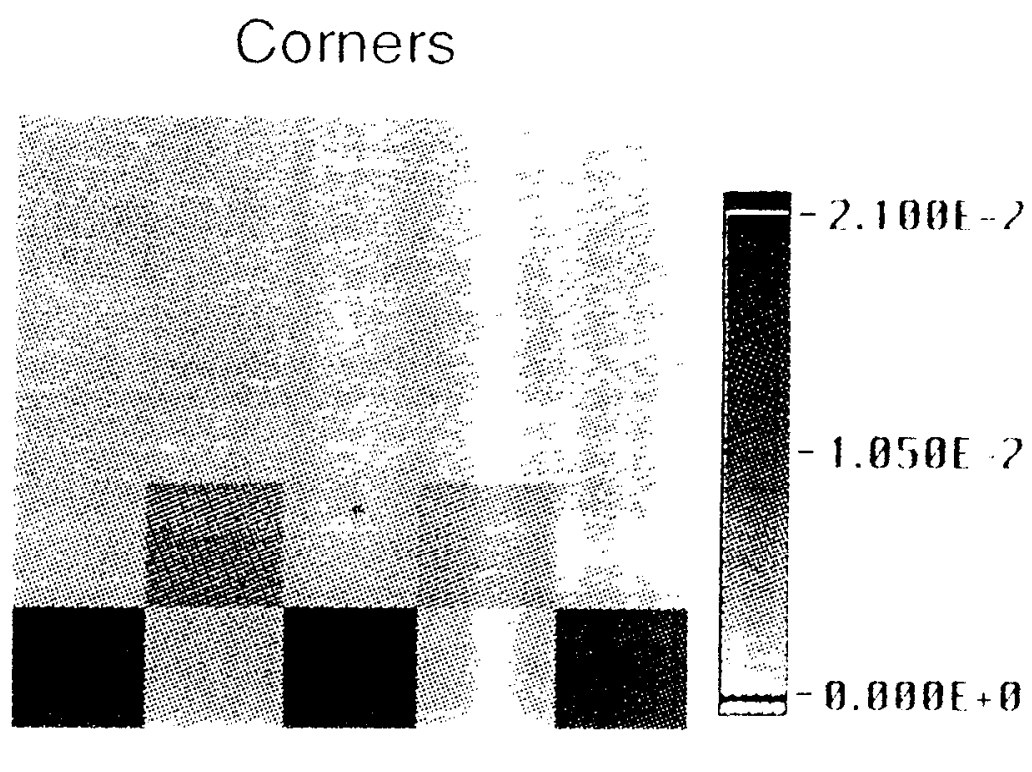

Others
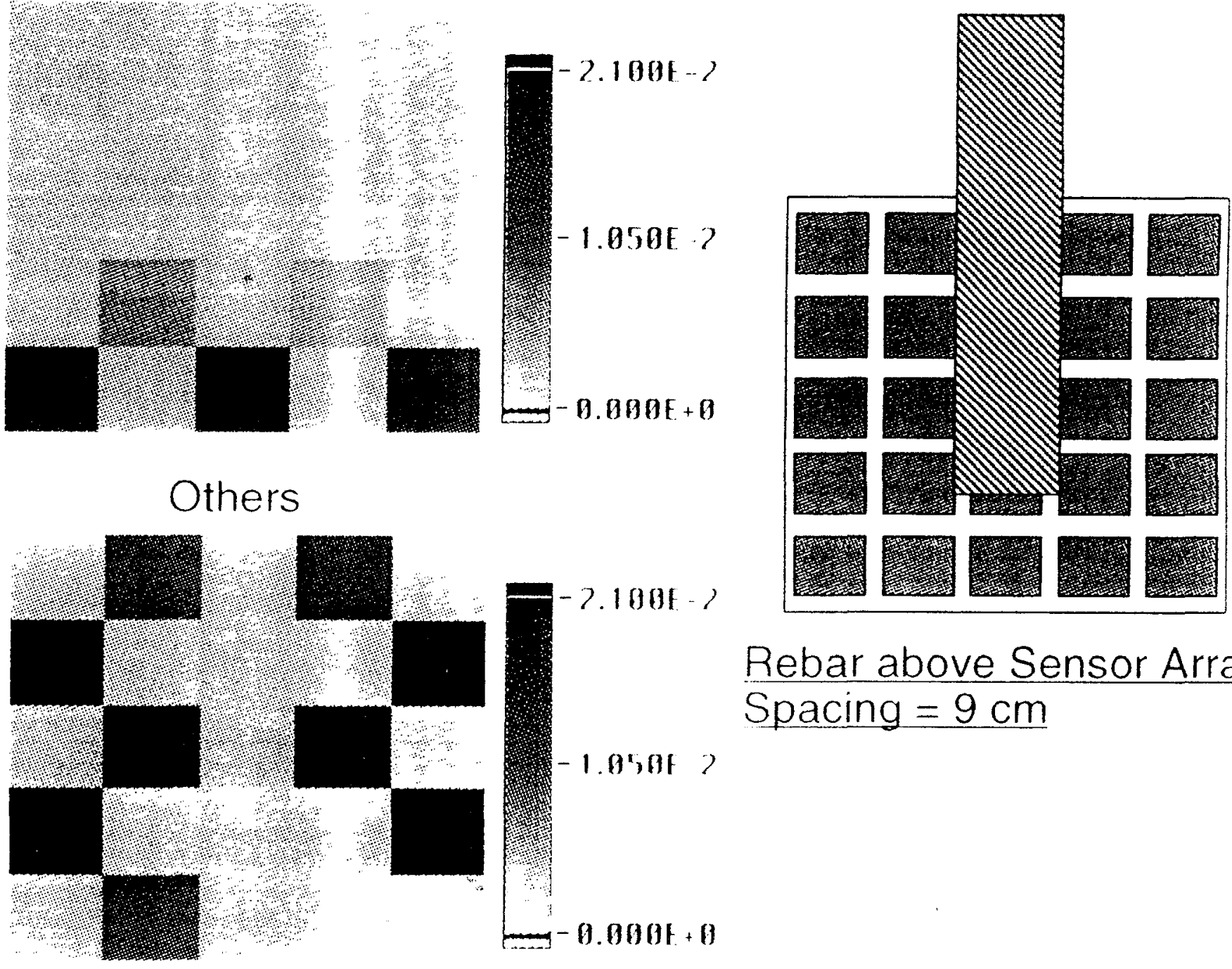

Rebar above Sensor Array Spacing $=9 \mathrm{~cm}$ 


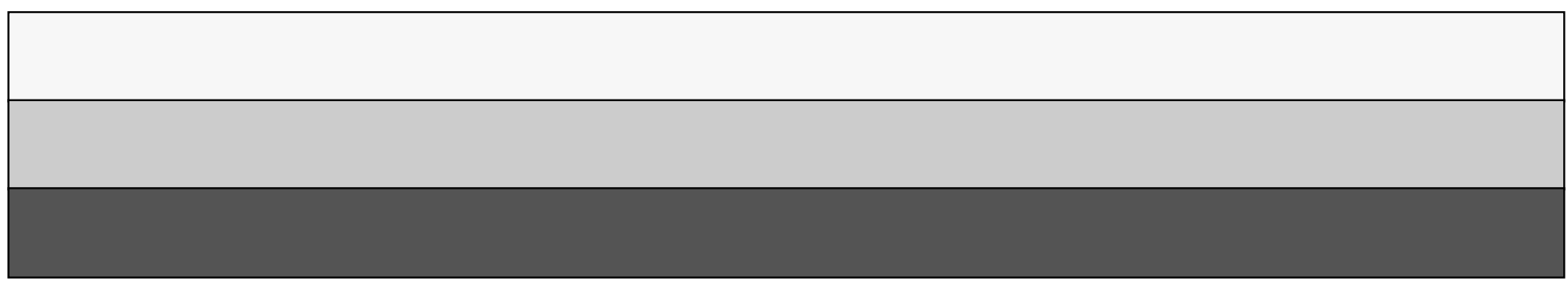

